Revista Luna Azul - ALGUNAS REFLEXIONES SOBRE EL ANÁLISIS TERRITOR... Página 1 de 17

\title{
ALGUNAS REFLEXIONES SOBRE EL ANÁLISIS TERRITORIAL Y LA ADMINISTRACIÓN DEL MEDIO AMBIENTE EN COLOMBIA
}

\author{
Jorge Andrés Rivera Pabón \\ Administrador del Medio Ambiente, Universidad Tecnológica de Pereira, UTP. \\ Magíster en Geografía con énfasis en Ordenamiento Territorial. Convenio UPTC-IGAC \\ Profesor Universidad de Caldas. Departamento de Historia y Geografía. \\ jorgeandres.rivera@ucaldas.edu.co
}

Manizales, 2007-10-30 (Rev. 2007-11-28)

\section{RESUMEN}

En este artículo se presenta una revisión crítica de los enfoques conceptuales y metodológicos que sustentan los ejercicios de análisis territorial y la administración del medio ambiente desarrollados por las instituciones encargadas de la investigación y gestión de los conflictos y problemas ambientales en el país y a nivel regional, como son, entre otros: el Ministerio de Ambiente, Vivienda y Desarrollo Territorial (MAVDT), el Instituto Geográfico Agustín Codazzi (IGAC) y la Corporación Autónoma Regional del Risaralda (CARDER), siendo esta última citada por ser una de las corporaciones regionales más reconocidas en la gestión ambiental de carácter público.

Esta revisión crítica es particularmente útil, en la medida que permite plantear a partir de los aspectos positivos y la explicación de las limitaciones teórico-metodológicas de los enfoques utilizados por dichas instituciones, una propuesta académica complementaria de análisis territorial y administración del medio ambiente fundamentada en la disciplina geográfica y el aporte interdisciplinario de la antropología, sociología, psicología, economía, etc., que sirva para superar los enfoques funcionalistas, fisicalistas y organicistas asociados a las propuestas institucionales.

\section{PALABRAS CLAVE}

Conflictos ambientales, análisis territorial (funcionalismo, fisicalismo, organicismo), ordenamiento territorial, zonificación ambiental, administración del medio ambiente.

SOME REFLECTIONS ON THE TERRITORIAL ANALYSIS AND ENVIRONMENTAL ADMINISTRATION IN COLOMBIA

\begin{abstract}
This paper presents a critical review of conceptual and methodological approaches that support territorial analysis and environmental management developed by the institutions responsible for the research and management of environmental problems in the country and on a regional level, such as: Ministry of Environment, Housing and Territorial Development, Agustin Codazzi Geographic Institute and Regional Environmental Corporation of Risaralda, this last one included for being one of the most important public environmental regional authorities.
\end{abstract}

This critical review is particularly useful since it allows, from the positive aspects and the explanation of theoretical and methodological limitations of traditional approaches used by those institutions, to draw a complementary academic perspective of territorial analysis and environmental management based on geography and the interdisciplinary 
Revista Luna Azul - ALGUNAS REFLEXIONES SOBRE EL ANÁLISIS TERRITOR... Página 2 de 17

input from anthropology, sociology, psychology, economics, etc.., which serves to overcome the functionalist, physical, organic and ecological approaches associated with institutional proposals.

\section{KEYWORDS}

Environmental conflicts, territorial analysis (functionalism, physicalism, organicism), environmental management, land use and zoning.

\section{INTRODUCCIÓN}

El análisis territorial aplicado en la acción institucional de administración y gestión ambiental, se ha desarrollado tradicionalmente como un ejercicio funcional para los actores estatales comprometidos con esta gestión, con el fin de proveer soluciones a los problemas provenientes de la relación sociedad-naturaleza. Paralelamente, se ha consolidado tanto en las altas esferas de la planificación como en el imaginario de la gran mayoría del colectivo social, que los problemas ambientales son resueltos o atendidos con acciones o proyectos tecnológicos e ingenieriles, sin ninguna necesidad de modificar las amplias fuerzas condicionantes de naturaleza política, económica y cultural que actúan en el espacio geográfico y en las relaciones entre los grupos humanos y su entorno. De igual manera, la contribución potencialmente significante de las acciones y pensamiento "no científico" de diversos actores no pertenecientes a la administración estatal, como por ejemplo las comunidades campesinas, negritudes, indígenas, entre otros, han sido notablemente desatendidas. Las dificultades enunciadas anteriormente se asocian, por lo general, con el enfoque positivista asumido no pocas veces en la acción "racional" de los funcionarios de la administración ambiental gubernamental.

A partir del reconocimiento de estas condiciones limitadas y restrictivas, evidenciadas en la forma como se realiza la gestión ambiental tradicional de los problemas asociados a la relación sociedad-naturaleza, se esboza, en primer lugar, cómo el análisis territorial se centra en estudios exhaustivos de tipo biofísico (zonas de potencial hídrico y balances hídricos, procesos geodinámicos, zonificación forestal, pérdida de biodiversidad, etc.), dejando relegada o dándole poco peso representativo a la disertación sobre las características que explican los procesos sociales $y$ sus causalidades históricas que son el factor detonador de los conflictos ambientales en el país (tenencia de la tierra y especulación del valor del suelo rural y urbano; relación entre el conflicto territorial armado, las migraciones forzadas, el destierro y los megaproyectos "estratégicos para el desarrollo económico"; la política "pública" de la vivienda vs. financiación privada y mercado de la vivienda; las implicaciones de la reestructuración de la economía global en los espacios geográficos locales y regionales, el desempleo y el empleo informal; las lógicas de construcción de asentamientos humanos marginales o la configuración territorial de barrios de "esfuerzo popular" en contraposición a la denominación de subnormalidad, etc.).

En segundo término, después de contemplar las razones y consideraciones enunciadas anteriormente, se presenta un replanteamiento del análisis territorial ambiental, compuesto por un enfoque amplío e integral. De esta forma, se describen las bases y localización disciplinaria de esta nueva propuesta que finalmente constituye los fundamentos para re-pensar y re-construir la Administración del Medio Ambiente desde su perspectiva tutelar: la interdisciplina.

1. ENFOQUES TEÓRICO-METODOLÓGICOS DEL ANÁLISIS TERRITORIAL AMBIENTAL EN EL MARCO DE LA PLANIFICACIÓN Y EL ORDENAMIENTO TERRITORIAL COLOMBIANO.

El análisis ambiental territorial en Colombia se ha circunscrito, usualmente, a las prácticas institucionales de manejo de los recursos naturales como parte de los ejercicios de planificación territorial. Con el propósito de conocer y analizar los conceptos y metodologías que sustentan el quehacer de esta práctica, se exponen a 
Revista Luna Azul - ALGUNAS REFLEXIONES SOBRE EL ANÁLISIS TERRITOR... Página 3 de 17

renglón seguido los enfoques teóricos y metodológicos del análisis territorial efectuado por la administración ambiental nacional y regional en el marco del Ordenamiento Territorial y el Ordenamiento Ambiental Territorial.

\subsection{Ministerio de Ambiente, Vivienda y Desarrollo Territorial (1)}

Este Ministerio, siendo el ente rector en la definición de la política ambiental y de sus directrices de ejecución, expone el análisis territorial de los conflictos ambientales en el marco de la gestión adelantada por las instituciones que integran el Sistema Nacional Ambiental; sin embargo, es menester señalar la preponderancia que tiene este aspecto dentro de la Ley 388 de 1997 (2).

En esta Ley de Ordenamiento Territorial Municipal el concepto que dirige la temática ambiental, es justamente el ordenamiento ambiental territorial, el cual se entiende como un conjunto de acciones estructuradas alrededor de las funciones ambientales específicas que cumple cada unidad del territorio, con el propósito de lograr que tales funciones estén en concordancia con la potencialidad natural de cada unidad, dentro de contextos locales, regionales y nacionales (3).

Tales acciones exigen, no sólo la identificación y espacialización de las unidades territoriales de acuerdo con su función ambiental, de los procesos y tendencias de apropiación y manejo de las mismas, sino la generación de instrumentos legales, económicos, sociales, políticos y administrativos que posibiliten dar un uso y desarrollo más adecuado a cada unidad y al territorio nacional en su conjunto.

De igual modo, interesa la definición de los problemas ambientales del territorio, señalando entonces que la contradicción entre el mantenimiento de los bienes y servicios que la naturaleza aporta a la sociedad y cualquier otro uso o actividad humana que implique transformación degradadora de la base natural, configura un conflicto ambiental.

En este sentido, el ordenamiento ambiental del territorio se plantea tres grandes propósitos en relación con los tiempos sobre los que actúa: hacia el futuro, la prevención de conflictos ambientales; en el presente, la resolución de conflictos ambientales; y en relación con el pasado, la reversión de procesos de deterioro ambiental.

Para estos diferentes propósitos es necesario identificar a los actores involucrados en cada situación, con el fin de determinar cómo alcanzarlo y qué responsabilidades tiene cada uno de ellos (4) . Singular importancia reviste el carácter prospectivo que debe comportar el ordenamiento ambiental del territorio, toda vez que la tarea de prevenir conflictos ambientales incide notablemente en el éxito de la gestión ambiental y aumenta su eficiencia. Para orientar y regular los procesos de ocupación, apropiación y uso del territorio y los recursos naturales, con la intención de garantizar su adecuado aprovechamiento y desarrollo sostenible, el Ministerio formuló los siguientes objetivos generales:

Figura 1. Objetivos del Ordenamiento Ambiental Territorial. 


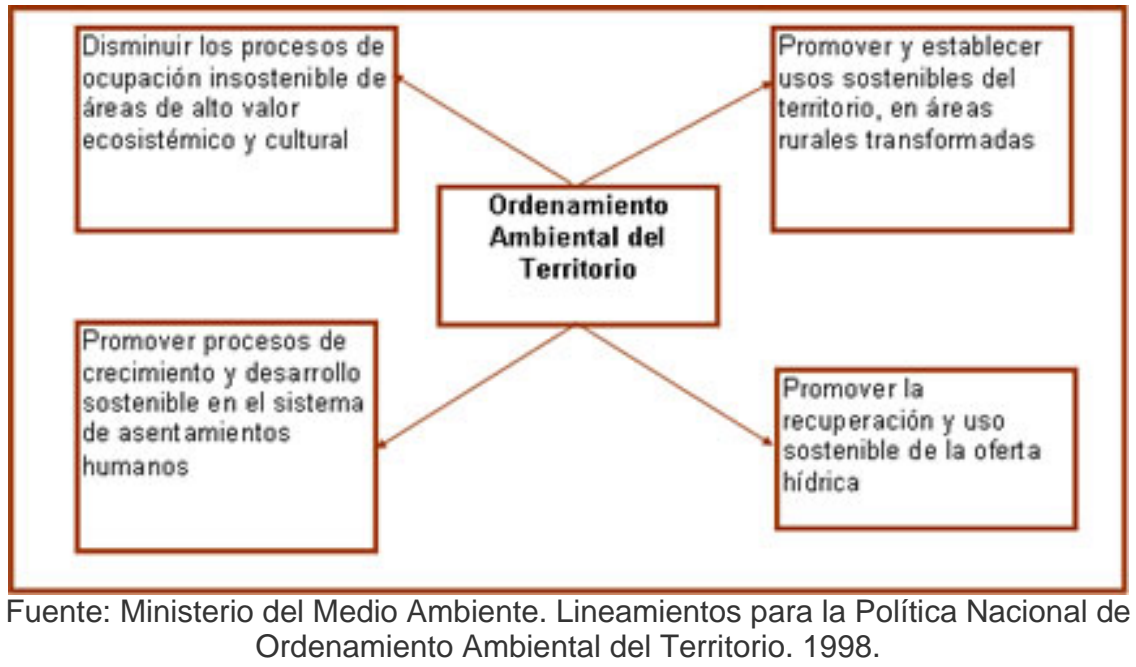

De esta forma, se percibe "al ordenamiento ambiental del territorio (O.A.T.) como "un componente fundamental, ineludible e indisociable del ordenamiento territorial (O.T.)" (5) . La orientación de los procesos de uso y ocupación del territorio deberá ser evaluada considerando los impactos e implicaciones en los ecosistemas; el Ordenamiento Ambiental Territorial suministra al Ordenamiento Territorial una síntesis de la estructura y dinámica de los ecosistemas, una valoración de los principales conflictos y potencialidades, y las diferentes propuestas de alternativas de uso, actividades y programaciones en el marco de la sostenibilidad. El punto de contacto de los procesos de O.T y O.A.T está en la planificación del uso del territorio, como factor básico para avanzar hacia el desarrollo sostenible (6) .

En conclusión, el ordenamiento ambiental del territorio es entendido por esta institución pública como un proceso técnico, administrativo y político que parte de la zonificación del uso adecuado del territorio con un enfoque ecosistémico o zonificación ambiental, el cual establece la compatibilidad de las actividades que se realizan en las diferentes unidades territoriales, con las características ecológicas, sociales y económicas de las mismas, que hagan sostenible el desarrollo desde el punto de vista ambiental. Dicha sostenibilidad está relacionada con el balance entre la capacidad ecológica de bienes y servicios ambientales de los ecosistemas y la presión de la demanda de éstos ejercida por la sociedad, oferta que define una capacidad de soporte con límites determinados por la productividad total del respectivo ecosistema natural, incluida su capacidad de resilencia, la protección y compensación que puede incorporar el hombre a través de tecnologías proteccionistas (7) .

\subsection{Instituto Geográfico Agustín Codazzi, IGAC.}

Por parte de este Instituto Geográfico, "el Ordenamiento Territorial es una política de Estado y un Instrumento de planificación, que permite una apropiada organización político- administrativa de la Nación, y la proyección espacial de las políticas de desarrollo social, económico, ambiental y cultural de la sociedad, garantizando un nivel de vida adecuado para la población y la conservación del ambiente" (8) .

Dentro de las funciones de la División de Ordenamiento Territorial del IGAC adscritas al área estratégica de Geografía, se encuentran:

1. Realizar investigaciones, diseñar metodologías y prestar asistencia técnica e interventorías en sistemas de información geográfica, como apoyo a la regionalización y el ordenamiento territorial.

2. Coordinar el suministro de información geográfica y el apoyo que el Instituto deba prestar para los procesos de ordenamiento territorial.

3. Participar en el proceso de validación de la información oficial con el fin de elaborar las bases y recomendaciones técnicas para el ordenamiento territorial, en coordinación con el Instituto de Hidrología, Meteorología y Estudios Ambientales, IDEAM, y el Instituto de Investigación e Información Geocientífica, 
Revista Luna Azul - ALGUNAS REFLEXIONES SOBRE EL ANÁLISIS TERRITOR... Página 5 de 17

Minero, Ambiental y Nuclear, INGEOMINAS, o la entidad que haga sus veces.

4. Apoyar técnicamente la evaluación de expedientes de titulación y la determinación de los límites de tierras de comunidades negras.

5. Producir y actualizar los mapas temáticos de resguardos indígenas y tierras de comunidades negras.

6. Efectuar el deslinde de las tierras que conformen resguardos indígenas, con fines político-administrativos y de planeación del desarrollo de las comunidades.

7. Apoyar el diseño y establecimiento de los lineamientos, normas, estándares, técnicas y símbolos de representación en mapas temáticos, y contribuir a su implementación.

8. Las demás funciones inherentes a la naturaleza de la dependencia y las que le sean asignadas por las disposiciones legales vigentes.

En cuanto a la relación del Ordenamiento Ambiental Territorial con el Plan de Ordenamiento Territorial, se establece a partir de la asignación de usos del suelo en áreas que requieran de un manejo especial, de acuerdo con las problemáticas, potencialidades o restricciones y en la búsqueda del desarrollo sostenible.

En consecuencia, para esta institución el ordenamiento ambiental territorial se hace imperioso, con el fin de "regular la utilización, la transformación y ocupación del espacio, de acuerdo con el desarrollo socioeconómico y en armonía con el medio natural y las tradiciones históricas y culturales" (9) .

\subsection{Corporación Autónoma Regional de Risaralda, CARDER.}

La CARDER como entidad del orden departamental dentro del Sistema Nacional Ambiental ha expuesto una conceptualización y propuesta metodológica de zonificación ambiental (10), con base en las directrices del Ministerio de Ambiente, Vivienda y Desarrollo Territorial para el Ordenamiento Ambiental Territorial.

En ella, se ha definido a la zonificación ambiental como la síntesis espacializada de áreas del territorio, en función de su interés ambiental. Esta propuesta técnica tiene como objeto la formulación de escenarios alternativos para el uso del territorio y como instrumento de jerarquización, priorización y concertación de planes, programas y proyectos. A su vez, se indica su utilidad para delimitar los espacios a conservar o ampliar por su interés natural, agrícola, forestal o paisajístico. De esta forma, posibilita distribuir espacialmente los usos del suelo y actividades a las que debe destinarse prioritariamente el mismo, señalando el uso principal o secundario, excluyente o alternativo de tales usos o actividades.

Figura 2. Esquema metodológico de zonificación ambiental

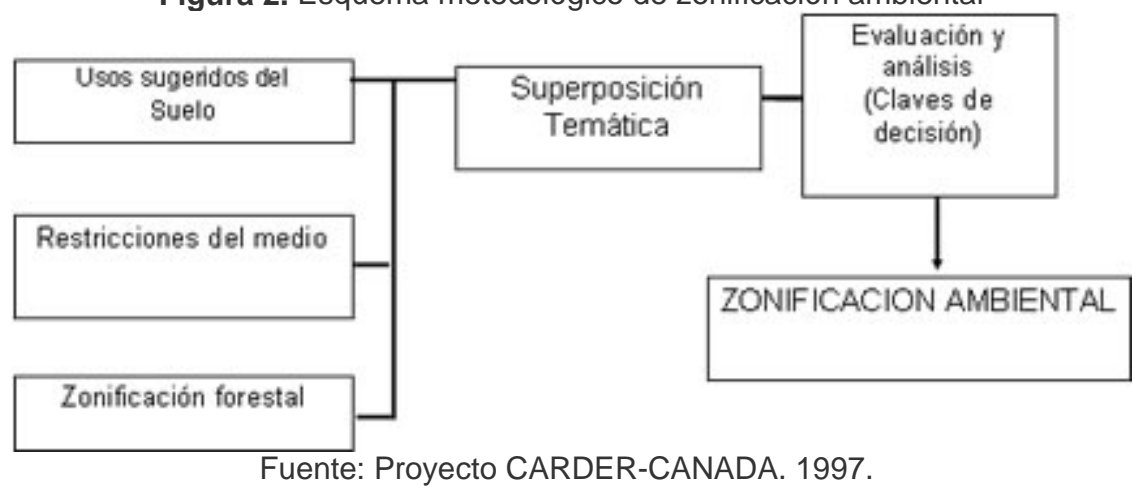

Como muestra el esquema anterior, para la determinación de la Zonificación Ambiental se requieren unas fases o etapas preliminares. En primera instancia, la definición de los Usos Sugeridos, que indican las recomendaciones en ciertas áreas del territorio de unas zonas que permiten establecer actividades compatibles, compatibles bajo ciertas condiciones y no compatibles, de acuerdo con las realidades biofísicas y socioeconómicas. 
Revista Luna Azul - ALGUNAS REFLEXIONES SOBRE EL ANÁLISIS TERRITOR... Página 6 de 17

Para su determinación se deben tener en cuenta los mapas de conflictos de uso, aptitud de usos de acuerdo al área de estudio y restricciones por amenaza. En lo socioeconómico se consideran las políticas de uso y tipo de ocupaciones.

Figura 3. Esquema metodológico de zonificación de usos sugeridos

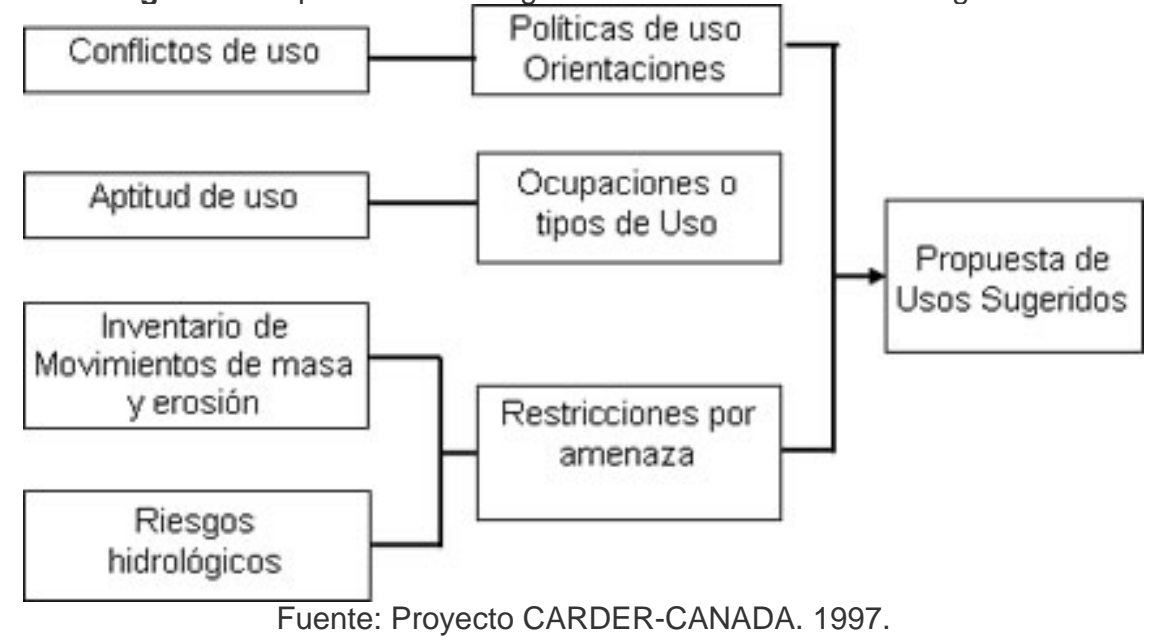

En segundo lugar, se estudian las zonas de restricciones de uso, las cuales se definen como aquellas áreas donde la ocupación del suelo está sometida a restricciones particulares por razones de seguridad pública. Esta restricción la determina, como lo demuestra la gráfica anterior, los movimientos en masa, el riesgo por inundación, entre otros factores de orden biofísico. De esta manera, su definición sirve para identificar y delimitar porciones del territorio en las que es necesario adoptar acciones para prevenir o mitigar el riesgo para la vida, la seguridad y la propiedad de los individuos y la colectividad.

En tercer término, se exhibe como elemento o insumo para la determinación de la zonificación ambiental, la zonificación forestal. Esta es una propuesta de delimitación de zonas que surge de definir, ordenar y espacializar áreas de cobertura (ecosistemas boscosos existentes) y suelos de aptitud forestal.

Figura 4. Esquema metodológico de zonificación forestal

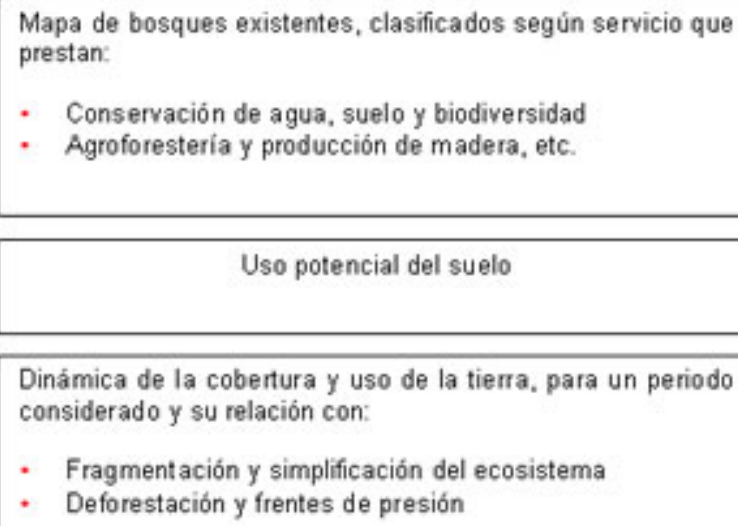

Uso potencial del suelo

Dinámica de la cobertura y uso de la tierra, para un periodo considerado y su relación con:

- Fragmentación y simplificación del ecosistema

- Deforestación y frentes de presión

\begin{tabular}{|c|}
\hline \multicolumn{1}{|c|}{ Restricción por amenaza } \\
\hline \multicolumn{1}{|c|}{ Zonificación del recurso hídrico } \\
\hline - Zonas aferentes a acueductos \\
- Zona de potencial hídrico subterráneo \\
\hline
\end{tabular}


Revista Luna Azul - ALGUNAS REFLEXIONES SOBRE EL ANÁLISIS TERRITOR... Página 7 de 17

Fuente: Proyecto CARDER-CANADA. 1997.

\title{
1.4 Consideraciones críticas del análisis territorial y el ordenamiento territorial
}

\begin{abstract}
"El espacio no es un ente de razón, ni algo a inventarse por cada planificador: existe y tiene vida propia; antes que nada hay que empezar por conocer como son realmente" (Jean Labasse, 1979) (11) .
\end{abstract}

A manera de análisis crítico, es preciso señalar que estas propuestas comparten ciertos aspectos positivos y una serie de limitaciones que deben ser objeto de reflexión. Así, se exhiben como puntos positivos los siguientes:

- La relación entre el ordenamiento territorial y la gestión ambiental ha permitido la inclusión como principio rector y tema relevante en la agenda de la planificación los temas ambientales. En este sentido, "el Ordenamiento Territorial propugna por la protección, conservación y aprovechamiento sustentable de áreas y ecosistemas de importancia para los municipios, al igual que por el control de los procesos de degradación ambiental generados por los sistemas productivos (industria, comercio, etc) y los asentamientos humanos, con miras a lograr mejores condiciones de vida para la población" (12) , de acuerdo a lo expuesto por las instituciones públicas reseñadas.

Figura 5. Objetivos del Desarrollo Territorial y el Plan de Ordenamiento Territorial en

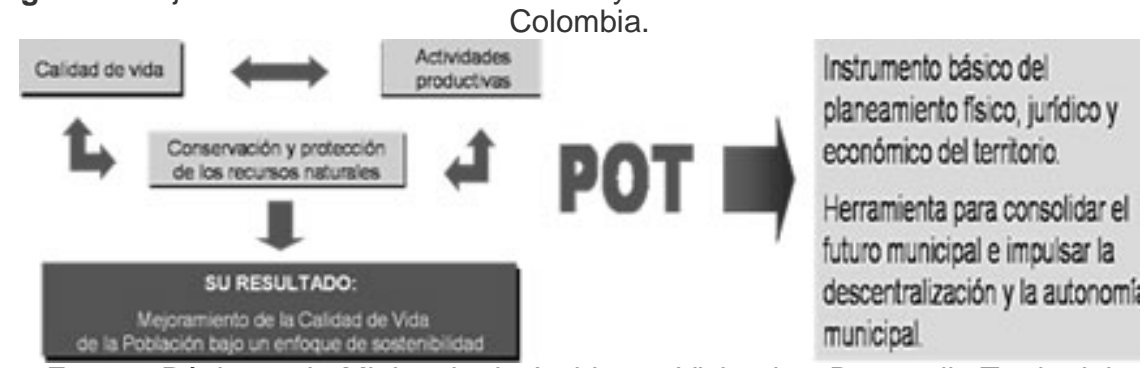

Fuente: Página web, Ministerio de Ambiente, Vivienda y Desarrollo Territorial (www.minambiente.gov.co)

- La implementación de un marco legal en el país sobre Desarrollo Territorial que permite la ejecución de análisis técnicos, los cuales soportan la definición de instrumentos de planificación y sus respectivos procesos de gestión, al igual que los mecanismos de financiación.

- El papel más importante de las zonificaciones es su aplicación futura, ya que pueden constituirse en documentos técnicos que soporten la planificación territorial municipal y también en un elemento que suscite la coordinación de planes, programas y proyectos de inversión o presupuesto para la gestión ambiental del territorio.

- A la par, se constituyen en una base de datos de información primaria y secundaria necesaria como herramienta de conocimiento para la planificación ambiental, en la cual se encuentran consignadas las características biofísicas y socioeconómicas presentes en el espacio geográfico. Esto permite a los administradores ambientales comprender, generar propuestas y participar en los ejercicios de gestión territorial.

De otra parte, es necesario adelantar la identificación de las debilidades o limitaciones resultantes de los enfoques teórico-metodológicos en los que se apoya el análisis territorial ambiental:

- Una restricción encontrada en la conceptualización y comprensión del análisis ambiental es la aproximación realizada a partir de la sumatoria de diversos 
subsistemas, entre los que se encuentran los subsistemas biofísico, socioeconómico y cultural, faltando una mayor preeminencia de la interacción o relación que se da entre todos ellos; así, se percibe un enfoque multidisciplinario, sumatoria de disciplinas, y no un énfasis interdisciplinario, que permitiría la interconexión discursiva, conceptual y metodológica, lo cual haría más incluyente las estrategias sociales a la hora de definir soluciones plausibles a los diferentes conflictos ambientales ocurridos en diversos ámbitos espacio temporales. De este modo, se percibe el enfoque fragmentado del análisis territorial caracterizado por la agregación de elementos biofísicos y sociales como dos subsistemas separados en la realidad "ambiental".

- Se constata el enfoque funcionalista del Ordenamiento Territorial al exponer que éste posibilita racionalizar la intervención sobre el territorio, propiciando su desarrollo y aprovechamiento ambiental sostenible a través de una decisión política administrativa, sustentada en un ejercicio técnico (zonificaciones).

Este último concepto señala claramente el interés por situar al "desarrollo sostenible" y a la "zonificación ambiental", como el criterio y la técnica que permitirán lograr per se, el "uso ordenado del territorio y la distribución armónica de los asentamientos humanos y las actividades productivas"; desconociendo que el espacio geográfico evidencia las huellas históricas del conflicto social, las contradicciones y la anarquía permanente del "orden territorial" promovido por los objetivos del "desarrollo económico sostenible", los cuales determinan y definen mediante la planificación territorial actual, en gran medida, la localización espacial de algunas actividades económicas en zonas de protección o con "aptitud y potencialidad agrícola y forestal", en áreas definidas de conservación y no para ser urbanizadas o destinadas a otro tipo de servicios (comerciales, equipamientos privados como bodegaje, parques tecnológicos e industriales, estaciones de gasolina, etc.).

- La mirada fisicalista y naturalista obedece a la preponderancia de lo biofísico sin contar de manera suficiente con las relaciones de causalidad y correspondencia que se da entre lo humano y lo entendido como "lo natural".

- La visión organicista relaciona los conflictos socio-naturales (ambientales) con patologías que rompen la "normalidad, armonía, orden y equilibrio de la naturaleza". Desde esta perspectiva, el análisis territorial se utiliza para "diagnosticar los síntomas del daño ambiental", siendo recurrentemente señaladas las comunidades caracterizadas por asentamientos humanos marginales como "agentes o vectores" que rompen el balance natural del ambiente. En correspondencia, las soluciones o "curas" a este tipo de anomalías territoriales se superan con el aprovechamiento racional del territorio, es decir, mediante el desarrollo sostenible; solicitud que no cumple y permite dicha población.

- De otro lado, es posible afirmar que el ordenamiento ambiental territorial presenta una hibridación en sus técnicas y estrategias un tanto contradictorias. En primer lugar, exhibe un carácter indicativo con el propósito de lograr el "aprovechamiento racional en el uso de la tierra", para lo cual se considera la superposición temática de factores como las potencialidades, restricciones y conflictos ambientales, etc.; que permiten obtener la definición de espacios compatibles, compatibles bajo ciertas condiciones y no compatibles. En segundo lugar, expone un enfoque normativo rígido ejemplificado claramente en su esencia final de formalizar instrumentos legales para el uso, ocupación y transformación del espacio en función del modelo de desarrollo económico, y en tercer lugar, presenta el carácter voluntarista y facilitador típico de la geografía del "laisser-faire" (13), en donde el liberalismo con sus nociones de competitividad, eficiencia y racionalidad económica "suscitan espontáneamente la armonía espacial perfecta", en virtud del buscado desarrollo económico "sostenible" y la "buena" calidad de vida de toda la población.

En este sentido, se puede inferir que el "Ordenamiento Ambiental Territorial" es un mecanismo e instrumento técnico que intenta hacer compatible un territorio con las 
Revista Luna Azul - ALGUNAS REFLEXIONES SOBRE EL ANÁLISIS TERRITOR... Página 9 de 17

exigencias trazadas por el sistema socioeconómico actual de internacionalización y apertura económica; por esta razón, se define por parte de la institucionalidad ambiental, el requerimiento imperioso de orientar el énfasis en el "rediseño de la organización espacial" (14), en la eficiencia de los equipamientos, de las redes de servicios y sistemas que estructuran los diferentes territorios. En otras palabras, el orden espacial lo determina "lo funcional que se pueda ser para el sistema económico" y la capacidad de atracción hacia la inversión; de esta forma, la espacialidad configurada por los procesos sociales, económicos y culturales tradicionales y "espontáneos", es categorizada como disfuncional y "desordenada" para este sistema, hasta tanto no se pueda obtener beneficios de sus condiciones y características (diversidad ecológica, cultural, posibilidades en cuanto a sus servicios ambientales, etc.).

\section{APORTES CONCEPTUALES PARA LA RE-CONSTRUCCIÓN INTERDISCIPLINARIA DE LA ADMINISTRACIÓN DEL MEDIO AMBIENTE (15)}

En seguida se presenta una conceptualización que posibilita una lectura y comprensión más amplia del fenómeno territorial de "utilización del espacio geográfico" o en palabras del geógrafo Jean Labasse "la modificación del marco de la existencia" (16) .

\subsection{Bases y localización disciplinaria de la propuesta de análisis territorial ambiental.}

Esta propuesta de análisis territorial ambiental integra el discurso teórico concordante de varias disciplinas que encuentran puntos e intereses comunes en su quehacer académico en torno a la temática ambiental. Este proceso se da a partir del encuentro de disciplinas y subdisciplinas de las ciencias sociales y naturales; dicha posición es respaldada por autores como Benton y Redclift, los cuales enfatizan "que la investigación sobre el medio ambiente se apoya y ocurre en diferentes puntos de convergencia entre varias disciplinas" (17) . Del mismo modo, si el objetivo de la reevaluación del análisis territorial es comprender la complejidad que caracteriza las prácticas, relaciones e intereses humanos sobre y con el ambiente, entonces su labor debe fortalecer la investigación social.

Esta perspectiva interdisciplinaria, de igual manera, proveerá y permitirá tener los elementos y criterios necesarios para la resolución de los problemas inscritos en el marco de la administración y gestión ambiental. Esta propuesta se interesa, por lo tanto, en examinar e interpretar la génesis y procesos que determinan los conflictos ambientales, para esto es necesario, en primera instancia, reconocer que los criterios centrales de explicación no podrían ser, exclusivamente, aquellos ligados al medio físico-biótico, lugar en el cual se producen y "expresan" dichos conflictos. En este sentido, se asume un enfoque basado en una concepción integral de la relación sociedad-naturaleza; fundamentada en el hecho de que no existe oposición real en esta dupla de conceptos, sino que, por el contrario, existe una plena identidad en ellos (18) .

Figura 6. Influencias disciplinarias en la propuesta de análisis territorial y de la administración ambiental desde una perspectiva geográfica 


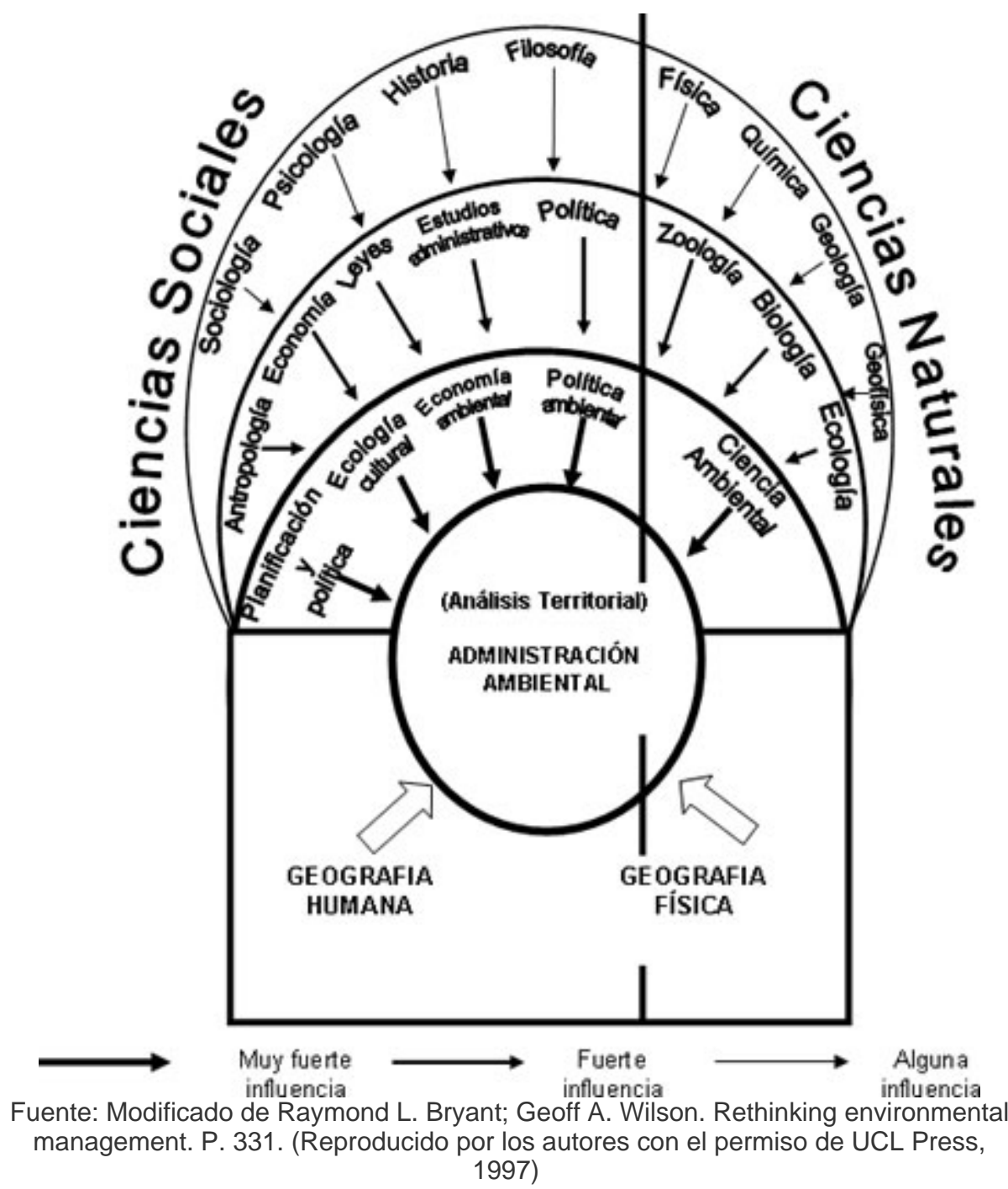

Sin embargo, es importante advertir que en este diagrama se exceptúan otras subdisciplinas que son importantes en este nuevo enfoque (19). Entre éstas se encuentran la geografía histórica, historia ambiental, economía y ecología política, ecología urbana, ecología cultural, etc. De esta forma, es fundamental introducir los conceptos elaborados por ellas en la construcción de una nueva visión del análisis territorial aplicado para los problemas ambientales.

Este enfoque sugiere también una mayor contribución de las ciencias sociales en el proceso de re-evaluación del análisis territorial (20) con el propósito de dilucidar y adquirir mayor entendimiento sobre la complejidad que caracteriza a los factores políticos, económicos y culturales que se involucran en la génesis y conformación de los problemas ambientales (21) .

Justamente, la pertinencia de las ciencias sociales permitirá consolidar y ampliar la participación de la geografía humana en el análisis territorial de los problemas ambientales, como también en su gestión, ya que hasta el momento, su contribución ha sido poco atendida (22), teniendo en cuenta su considerable desarrollo conceptual alrededor del tema del uso y manejo dado por el hombre al ambiente. Para superar este punto de partida, se presentan a renglón seguido diversos ejemplos que ilustran el aporte que podría prestar la geografía humana en esta propuesta de análisis territorial ambiental.

Primero, el trabajo fundado en la geografía económica, la cual explora los vínculos 
existentes entre varias categorías, como son el tiempo, el espacio y la rápida mutación de la economía global capitalista. Esta temática ha sido ampliamente desarrollada por autores como Corbrige (23) y Harvey (24) . Sus análisis revelan nuevas ideas en la comprensión de la dinámica acaecida entre la sociedad y la naturaleza, mediada por las relaciones socioeconómicas en diversas escalas.

La geografía económica aborda este estudio a través de cuestionamientos como: ¿han alterado los cambios económicos globales/neoliberales la manera como los diferentes actores sociales persiguen sus objetivos e intereses en la utilización del entorno y de administrar el ambiente? Otra consideración se origina en la siguiente incógnita: ¿Cómo puede la interpenetración de los negocios y los intereses estatales (por ej. empresas de carácter mixto) afectar diferencialmente la capacidad del Estado para actuar como mediador de "los intereses comunes o generales" en su papel de administrador de las relaciones entre la sociedad y el entorno?

En segundo lugar, las investigaciones adelantadas en geografía cultural también serían beneficiosas en la concepción de una visión y lectura más incluyente de los problemas o conflictos ambientales. De esta forma, se presenta como referencia ilustrativa el trabajo de Cosgrove y Daniels (25) , en temas como "la iconografía y percepción del paisaje". El objetivo que identifica este tipo de investigaciones es la comprensión de los cambios producidos en el medio por las huellas humanas, y la relación de ellas con la apreciación y valoración que se posea del "ambiente". Esta temática, por consiguiente, podría ser favorable para entender y dilucidar las interacciones entre las actitudes ambientales y las experiencias de manejo del entorno (26) .

En tercer lugar, se presenta el aporte potencialmente significante de la geografía histórica y la historia ambiental (27). Los estudios realizados dentro de estas subdisciplinas rompen con la visión tradicional de aproximación a los problemas de la relación sociedad-naturaleza desde un enfoque atemporal, incorporando las categorías fundamentales de las ciencias sociales. El objetivo de estas subdisciplinas es la comprensión de cómo las sociedades o grupos humanos han transformado la naturaleza para producir paisajes culturales; siendo un pilar conceptual el planteamiento de paisaje en términos de conjugación de temporalidades articuladas por la historia social (28) .

En cuarto lugar, los progresos teóricos y prácticos en geografía política pueden concurrir en la explicación de los nexos existentes entre los procesos políticos y la forma en que utilizamos y transformamos el ambiente. Por ejemplo, las investigaciones elaboradas por Agnew y Corbridge (29) y O'Tuathail (30), entre otros, exploran las conexiones que emergen de las relaciones de poder, espacio y territorialidad. Así, se logra superar la visión tradicional de espacio político, propiciando un enfoque a favor de una lectura más incluyente de cómo los actores estatales y no estatales interactúan en la creación y uso del espacio político. Estos trabajos tienen claras implicaciones para la re-evaluación del análisis territorial ambiental, ya que revelan las relaciones cercanas entre política, territorialidad y acceso a los recursos naturales. (Ej., Peluso (31) y Bryant (32)).

De igual modo, las propuestas de la ecología política se encuentran próximas a los nuevos intereses del análisis territorial de los problemas ambientales en razón de sus objetivos. Entre ellos está la búsqueda de un conocimiento de la economía política asociada a la interacción ambiente-sociedad humana. En esta perspectiva de análisis, se plantea cómo los problemas ambientales son resultado de las relaciones y factores sociales y económicos (por ejemplo, las consecuencias ambientales de la forma en que se toman las decisiones en las familias, la distribución y tenencia de la tierra, las políticas estatales); es decir, bajo esta óptica el examen técnico y local no es suficiente para proporcionar soluciones ambientales (Peet y Watts (33) ).

Las preguntas de investigación de esta área temática se concentran en la indagación de las fuerzas políticas que determinan el acceso y control de (a) los recursos naturales y su correspondencia con el cambio ambiental; como también en la interpretación del significado de la producción, uso y distribución de estos recursos (34) . La ecología política (35) tiene, entonces, gran pertinencia en la interpretación del papel que juegan las relaciones de poder en los cambios presentes de las condiciones ambientales. 
En definitiva, los argumentos planteados con anterioridad ilustran cómo un nuevo enfoque de análisis territorial, está estrechamente relacionado con las perspectivas expuestas por la geografía. Además, los interrogantes sobre el espacio ayudan a explicar la labor central de la geografía en el análisis territorial de los conflictos ambientales y las alternativas de gestión. Sobre todo, se establece la pregunta: ¿cómo están expresadas espacialmente las prácticas y políticas de uso del entorno por parte de los diversos actores sociales?

Como lo ha señalado MacNeill (36) el "espacio es una importante categoría en el estudio de los problemas ambientales" $y$, por consiguiente, de las soluciones propuestas para aquellos problemas. Aún si el campo de la administración ambiental, y particularmente, los ejercicios de análisis territorial, han reconocido durante mucho tiempo la jerarquía de la categoría espacio, es oportuno asimilar la gran riqueza de la literatura existente sobre geografía humana, específicamente aquella que versa sobre los aspectos socioculturales, económicos y políticos ligados a los cuestionamientos espaciales.

Mientras el análisis territorial ambiental está, en consecuencia, claramente relacionado con la geografía (Figura 6), no cabe duda que también necesite de la aportación de otras disciplinas. Si las preguntas en torno a lo "espacial" acentúan el rol central de la geografía en la comprensión y gestión de los conflictos ambientales, esta tarea teóricopráctica necesita de igual manera, ampliar la apreciación de los procesos económicos, políticos y culturales, con el aporte de algunos trabajos construidos desde la economía, las ciencias o estudios políticos y la antropología. Por ejemplo, para entender las actividades de los diferentes actores en el ambiente, es necesario apreciar cómo las relaciones de poder permiten o restringen aquellas actividades; siendo este un tema central en la literatura de los estudios políticos (Garner (37) ).

De manera similar, los trabajos realizados en economía pueden ayudar a clarificar las diferentes visiones que existen para abordar la introducción de los recursos naturales en la teoría económica. En primer lugar, se presenta una perspectiva desde la economía ambiental ortodoxa de inclusión de los servicios que presta la naturaleza como bienes transferibles en el mercado (Bromley (38) ); de otro lado, se exhibe un enfoque económico que argumenta cómo los servicios ambientales que presta la biosfera (procesos naturales como la asimilación de desechos, los ciclos biogeoquímicos, el disfrute estético del paisaje, etc.) no son cuantificables como un recurso de capital; sin embargo, platean la asignación de un "valor" indirecto de estos servicios ambientales, en razón al costo derivado (uso de tecnología, impuestos, etc) de la reducción de la contaminación de un recurso natural a estándares permitidos (Jacobs (39) ).

Las investigaciones en antropología, mientras tanto, están abriendo el camino a la interpretación de la dimensión cultural incorporada en los análisis territoriales de carácter ambiental; esto se manifiesta en el estudio de la correlación entre ciertas normas culturales y "Tabús", y su incidencia sobre algunas prácticas realizadas por diferentes actores sociales (Reichel-Dolmatoff (40) ).

Astrid Ulloa (41) pone de manifiesto la retroalimentación de los discursos provenientes de diversas subdisciplinas en torno al estudio de la relación naturaleza y ambiente, en particular en las actuales tendencias teóricas antropológicas. Así, destaca dos vertientes de aproximación. La primera que se puede denominar etnoecología, la cual está ligada al replanteamiento de las categorías duales (naturaleza y cultura) a la luz de los conocimientos raizales. En esta tendencia, se pueden localizar los estudios sobre prácticas locales basadas en categorías conceptuales y en los sistemas de conocimiento de las comunidades (indígenas, campesinas, urbanas, etc.) y los estudios sobre mundos híbridos tanto a nivel tecnológico como social.

La segunda tendencia se centra de manera general, en las interrelaciones de las prácticas locales con contextos amplios en el ámbito socioeconómico y político. Es aquí donde se podría ubicar la antropología del medio ambiente y la ecología política antropológica. Esta forma de abordar la temática, ha transformado la concepción de comunidad cerrada para tener en cuenta los diferentes actores que están interrelacionados en lo ambiental. 
La ecología cultural ha proveído conocimiento al análisis territorial integral, enriqueciendo la capacidad analítica del proceso. La ecología cultural ha centrado su atención durante mucho tiempo, en el manejo que las comunidades locales hacen del medio ambiente en que viven. Dentro de esta orientación, se ha producido un cambio tanto en sus objetos de estudio como en las bases epistemológicas que han servido para sustentar sus apreciaciones. De tal manera que se ha pasado de estudiar los sistemas productivos y la capacidad adaptativa a su entorno, de comunidades aisladas geográficamente, con base en los conceptos de la ecología; a estudiar en segundo término, grupos sociales en transición, es decir, aquellos que están siendo incorporados a la economía mundial. En esta etapa analítica, se ha considerado la existencia y mediación de otros aspectos sociales distintos a la mera supervivencia en el medio natural (manejo del entorno o administración de su ambiente), como puede ser el caso de la reproducción de clases sociales y la influencia de mercados externos, etc (42) .

Como se esbozó con anterioridad, la figura 6 sugiere algunas subdisciplinas que confluyen en el desarrollo del análisis territorial de los problemas ambientales (aunque necesita ser reiterado que no todas se anotaron en la figura). Desde este punto de vista, la política ambiental representa una útil (y hasta ahora subutilizada) fuente de conocimiento de la manera en la cual los heterogéneos problemas ambientales y actores, pueden relacionarse con los procesos políticos configurados en diferentes ámbitos y escalas.

Esta subdisciplina de los estudios o ciencias políticas proporciona una mirada a la naturaleza cambiante de las políticas ambientales trazadas por el Estado; de igual forma asume un análisis del pensamiento o filosofía verde de los partidos políticos y los diversos grupos de la sociedad civil (Wapner (43) ). Tales investigaciones podrían ser de gran ayuda para enfatizar y abordar con mayor precisión, el análisis de los intereses y objetivos políticos de los diferentes actores estatales y no estatales comprometidos en el manejo del ambiente.

\section{BIBLIOGRÁFIA}

- ANDRADE, Ángela. Ordenamiento Ambiental Territorial y Gestión Ambiental. En: Ministerio del Medio Ambiente - CIDER. El Ordenamiento Ambiental del Territorio. Panel de Expertos. Memorias. Sasaima, 1996.

- BIRCH, J. W. Geography and resource management. Journal of environmental management 1, 3-11. 1973.

- BRYANT Raymond; L.; WILSON Geoff. Rethinking environmental management. Department of Geography, King's College London, Strand, London, En: Progress in Human Geography 22, 3. 1998.

- CARDER - CANADÁ. Como se puede elaborar una propuesta de planificación territorial. Pereira, 1997.

- CARDER - CANADÁ. Planificación Territorial del Río Risaralda. Propuesta para una gestión del desarrollo sostenible. CARDER, CORPOCALDAS, ACDI, TECSULT. Pereira, 1997.

- CEPAL. Modelo de Gestión Ambiental a Nivel Municipal. Santiago de Chile, 1994.

- CORAGGIO, José Luis. Sobre la especialidad social y el concepto de región. CEPAL. Santiago de Chile. 1983.

- COSGROVE, D. And Daniels, S.J., editors. The iconography of landscape. Cambridge University Press. Cambridge. 1994.

- DNP y otros. Fundamentos Sobre el Ordenamiento Territorial como Instrumento de Planificación. Bogotá, 1996.

- HARVEY, David. The nature of environment: the dialectics of social and environment change. In Milliband, R. and Panitch, L., editors. Real problems-false solutions: socialist register. London: Merlin Press, 1993.

- JACOBS Michael. Economía Verde. Oxford: Blackwell. 1994.

- LABASSE, Jean. La organización del espacio. Elementos de geografía aplicada. Paris. Ed. HERMANN, Editeurs des Sciences et des Arts. 1979.

- MÁRQUEZ, Germán. Consideraciones Básicas Sobre Ordenamiento Ambiental y Ecosistemas Estratégicos en Colombia. Informe Ejecutivo - Ministerio del Medio Ambiente. Bogotá, 1997. 
- MINISTERIO DEL MEDIO AMBIENTE, OFICINA ASESORA DE ORDENAMIENTO AMBIENTAL. Bases Ambientales para el Ordenamiento Territorial Municipal en el marco de la Ley 388 de 1997. Bogotá, 1998.

- MOLANO Barrero Joaquín. Villa de Leyva. Ensayo de interpretación social de una catástrofe ecológica. Ed. FONDO FEN COLOMBIA. 1990.

- PALACIO Germán., ULLOA Astrid, Editores. Universidad Nacional, Sede Leticia; IMANI, ICANH, COLCIENCIAS. Repensando la Naturaleza. 2002.

- PEET Richard and Watts, M., editors. Liberation ecologies: environment, development and social movements. London: Routledge. 1996.

- REDCLIFT, M and BENTON, T., editors, Social theory and the global environment. London. Routledge. 1994.

- UTRIA Rubén Darío. La Sostenibilidad del Desarrollo Urbano. Santa fe de Bogotá, D.C., 1994.

\section{NOTAS}

1. Anteriormente designado Ministerio del Medio Ambiente.

2. MINISTERIO DEL MEDIO AMBIENTE, OFICINA ASESORA DE ORDENAMIENTO AMBIENTAL. Bases Ambientales para el Ordenamiento Territorial Municipal en el marco de la Ley 388 de 1997. Bogotá, 1998.

3. MÁRQUEZ, Germán. Consideraciones Básicas Sobre Ordenamiento Ambiental y Ecosistemas Estratégicos en Colombia. Informe Ejecutivo - Ministerio del Medio Ambiente. Bogotá, 1997.

4. CEPAL. Modelo de Gestión Ambiental a Nivel Municipal. Santiago de Chile, 1994.

5. DNP y otros. Fundamentos Sobre el Ordenamiento Territorial como Instrumento de Planificación. Bogotá, 1996.

6. ANDRADE, Ángela. Ordenamiento Ambiental Territorial y Gestión Ambiental. En Ministerio del Medio Ambiente - CIDER. El Ordenamiento Ambiental del Territorio. Panel de Expertos. Memorias. Sasaima, 1996.

7. UTRIA, Rubén Darío. La Sostenibilidad del Desarrollo Urbano. Santa fe de Bogotá, D.C., 1994.

8. Dentro de las actividades asignadas por el Estado Colombiano al IGAC en sus líneas estratégicas de investigación y desarrollo, se encuentra la Planificación y Ordenamiento Territorial. De esta forma se define que el IGAC tiene como Misión: "Cumplir el mandato constitucional referente a la elaboración y actualización del mapa oficial de la República de Colombia, desarrollar las políticas y ejecutar los planes de gobierno nacional en materia de Cartografía, Agrología, Catastro y Geografía, mediante la producción, análisis y divulgación de información catastral y ambiental georreferenciada, con el fin de apoyar los procesos de planificación y ordenamiento territorial". Justamente, esta última parte de la Misión Institucional, debe ser el mayor aliciente para que se realicen los más grandes esfuerzos investigativos sobre planificación y desarrollo territorial en bien del país, y aunque estos esfuerzos son ingentes, no existe otra entidad ni pública ni privada que pueda dar respuesta integral a esta necesidad Nacional. Véase esta información en: www.igac.gov.co

9. Ley de Ordenamiento Territorial Municipal 388 de 1997; Artículo 5.

10. La conceptualización y metodología de zonificación ambiental reseñada en este artículo como ejercicio adelantado por la CARDER es la inscrita en el documento "Como se puede elaborar una propuesta de planificación territorial: Experiencia en el territorio del río Risaralda". Proyecto CARDER-Canadá. Propuesta para una gestión del desarrollo sostenible. CARDER, CORPOCALDAS, ACDI Y TECSULT. Pereira, 1997. Es pertinente anotar que esta Corporación Autónoma ha sido líder en este tema, y continua trabajando para superar las miradas reduccionistas de la Administración y Gestión Ambiental (Véase: http://www.carder.gov.co/)

11. LABASSE, Jean. La organización del espacio. Elementos de geografía aplicada. Paris. Ed. HERMANN, Editeurs des Sciences et des Arts. 1979. p. 7.

12. Véase los respectivos lineamientos señalados en las páginas web de las instituciones mencionadas (www.minambiente.gov.co; www. carder.goc.co; www.igac.gov.co).

13. LABASSE. Op. cit., p. 15.

14. Entendiendo en este contexto organización espacial como la distribución funcional de las actividades a través del espacio, para corresponder a los 
intereses particulares monopólicos de acumulación de capital; muy en contravía a lo explicitado por Coraggio como Organización Espacial, "cuando una configuración es sostenida por un proceso social que la refuerza y conserva o cuando es producto de actos voluntarios en función de ciertos objetivos conscientes". Citado con base en: CORAGGIO, José Luis. Sobre la especialidad social y el concepto de región. CEPAL. Santiago de Chile. 1983. P. 20.

15. Este acápite está inspirado y elaborado principalmente, a partir del análisis del texto escrito por: Raymond L. Bryant; Geoff A. Wilson. Rethinking environmental management. Department of Geography, King's College London, Strand, London, En: Progress in Human Geography 22,3. 1998. p. 321-343.

16. LABASSE. Op. cit., p. 17.

17. BENTON, T. and REDCLIFT, M. Introduction. In REDCLIFT, M and BENTON, T., editors, Social theory and the global environment. London. Routledge. 1994. p.11., citado por: Raymond L. Bryant; Geoff A. Wilson. Op, cit. p. 331,332.

18. Por esto, los procesos ecológicos no desaparecen al interior de una formación social, ni tampoco es posible encontrar sociedades a-espaciales. De manera similar, las categorías espacio y tiempo no deben separarse en ningún proceso de conocimiento donde se pretenda comprender los conflictos ambientales resultantes de las actividades humanas. La trascendencia de los hechos se plasma en el espacio y permanece como formas, garantizando así una especialización en el tiempo. De esta manera, la comprensión de los conflictos ambientales se da a partir de la lectura del libro en que se escriben, el paisaje. Citado con base en: MOLANO, Barrero Joaquín. Villa de Leyva. Ensayo de interpretación social de una catástrofe ecológica. Ed. FONDO FEN COLOMBIA. 1990. p. 12.

19. El esquema indica las disciplinas incorporadas al análisis territorial. Asimismo, el grado de influencia lo refleja la amplitud o ancho de la flecha; además, es necesario manifestar que este esquema no muestra la situación inversa, es decir, la naturaleza y magnitud de la influencia de la administración ambiental sobre las diferentes disciplinas y subdisciplinas citadas.

20. Con base en las propuestas realizadas por diversos académicos y ambientalistas se ha constatado un cambio en torno al papel de las ciencias sociales en la temática ambiental; así se ha percibido, y lo muestra la figura, que la geografía física está perdiendo su papel central en este campo, en la medida que los temas sociales están tomando prioridad sobre los cuestionamientos de la esfera física. Citado por: Raymond L. Bryant; Geoff A. Wilson. Op. cit., p. 332

21. Igualmente, siguen cumpliendo un rol importante y con vigencia las ciencias naturales, especialmente la geografía física y las ciencias ambientales En este contexto es preciso hacer una distinción entre la administración ambiental y las ciencias ambientales. La primera se centra primordialmente en la explicación y comprensión de cómo los humanos interactuamos con el ambiente y con los demás seres humanos en nuestras prácticas de administrar el entorno (desde este punto de vista es esencialmente una ciencia social), en tanto que las ciencias ambientales centran su atención en la explicación e ilustración de los fenómenos y bases físicas de los cambios ambientales (demostrando ser una ciencia natural). Citado por: Ibíd. p. 332.

22. Esta disciplina, sin embargo, ha tenido influencia en algunas labores relacionadas con la gestión ambiental; argumento certificado con las contribuciones realizadas en las áreas de la planificación y la administración de recursos naturales. Estos aportes han sido plasmados en los textos de diferentes académicos de la geografía a nivel internacional; entre ellos, Compton plantea que "la planificación es absolutamente básica para la función de la administración ambiental, ya que considera la identificación de los objetivos y la selección de los medios y mecanismos para conseguirlos". En: Compton, P. Introduction. In Nath, B: Hens L; Compton, P. and Devuyst D; editors. Environmental management. Vol. 1. VUB Press. Brussels, 1993. p. 13-26. Otros autores son: O'Riordan T. Perspectives on resource management. London. 1971. Birch, J. W. Geography and resource management. Journal of environmental management 1, 3-11. 1973. Rees, J. Natural resources: allocation, economics and policy. "Second edition. London: Routledge. 1990. Selman, P. Environmental planning: The conservation and development of biophysical resources. Paul Chapman Publishing. London. 1992. Williams, R. H. European Union spatial policy and planning. Paul Chapman Publishing. London. 1996. Citado con base en: Ibid. p. 333.

23. Corbridge, S; Martin, R. and Thrift, N., editors. Money, power and space. Oxford 
University Press. Oxford. 1994. Citado con base en: Ibid. p. 333.

24. Harvey, D. The nature of environment: the dialectics of social and environment change. In Milliband, R. and Panitch, L., editors. Real problems-false solutions: socialist register. London: Merlin Press, 1993. Citado por: Ibid. p. 333.

25. Cosgrove, D. And Daniels, S.J., editors. The iconography of landscape. Cambridge University Press. Cambridge. 1994. Citado por: Ibíd. p. 334.

26. Las exploraciones efectuadas por Cosgrove sobre la "construcción social de la naturaleza" y "paisaje e identidad" proporcionan incógnitas acerca del consumo masivo y la percepción de la naturaleza, para evaluar los códigos ambientales modernos. De igual manera, este trabajo, intenta clarificar las amplias tendencias sociales y los cambios que se dan en las actitudes y labores ambientales. Citado por: Ibid. p. 334.

27. La diferencia entre la geografía histórica e historia ambiental, podría decirse, es mínima; a veces sólo perceptible en el estilo de su escritura y al mayor o menor carácter hermenéutico de los trabajos. En primera medida habría que mencionar que al observar la presencia de lo ambiental en las disciplinas históricas la primera constatación realizada es que ello ha estado más fuertemente insinuado, y a veces más explícitamente trabajado, el ámbito de una disciplina igualmente vieja como es la geografía. De esta forma, se podría afirmar, que la primera historia ambiental fue hecha por la disciplina geográfica. En segundo lugar, las dos profundizan en las relaciones entre lo temporal y lo espacial; siendo la diferencia el aspecto donde se centra la atención. La historia, enfatiza en el tema de la agencia humana, es decir, en el hecho de que los protagonistas y los antagonistas de su narrativa son siempre seres humanos, mientras que en la geografía histórica trabaja con mayor rigurosidad las variables exógenas del sistema ecológico. Citado con base en: Palacio Germán., Ulloa Astrid, Editores. Op. cit. p. 124-126.

28. Un trabajo geográfico relacionado con esta temática es el elaborado por Molano Barrero, Joaquín. Op. Cit. 1990.

29. Agnew, J. And Corbrige, S. Mastering space: Hegemonity, territory and international political economy. London: Routledge. 1995. Citado por: Raymond L. Bryant; Geoff A. Wilson. Op. cit., p. 334

30. O'Tuathail, G. Critical geopolitics. London: Routledge. 1996. Citado con base en: Ibíd., p. 334.

31. Peluso, N. L. Whose woods are these? Counter mapping forest territories in Kalimantan, Indonesia. Antipode 27, 383-404. 1995. Citado por: Ibid. p. 334.

32. Bryant, R. L. Political ecology: an emerging research agenda in third world studies. Political Geography 11, 12-36. 1992. Citado por: Ibid. p. 334.

33. Peet, R. and Watts, M., editors. Liberation ecologies: environment, development and social movements. London: Routledge. 1996. Citado por: Ibíd., p. 334.

34. Entre los interrogantes más comunes están: ¿cómo son apropiados y usados los recursos naturales por parte de los diversos actores sociales?; ¿cuáles pueden ser los tipos de conflictos ocasionados por dichos usos ambientales?; ¿existen relaciones de inequidad en el acceso a los recursos naturales, bienes y servicios ambientales? y ¿cómo podría la degradación ambiental afectar diferencialmente a las comunidades presentes en la sociedad? Citado por: Ibíd., p. 334.

35. La ecología política es otra subdisciplina de reciente formación; particularmente afianzada y desarrollada en el campo de la geografía. Hay que aclarar que este campo de estudio puede llevar a malentendidos, pues muchos de los trabajos en esta área tienen poco o nada que ver con la ecología. El nombre ecología política surgió de la simbiosis entre ecología cultural y economía política, ya que éstas dos subdisciplinas por separado obtuvieron resultados insuficientes para quienes querían combinar naturaleza y sociedad desde una perspectiva política. Esto se exhibe claramente en el texto pionero "La economía política de la erosión del suelo en los países en desarrollo" de Piers Blaikie, 1985. Citado con base en: Universidad Nacional, Sede Leticia; IMANI, ICANH, COLCIENCIAS. Repensando la Naturaleza. Palacio Germán., Ulloa Astrid, Editores. 2002. p. 130-131.

36. Raymond L. Bryant; Geoff A. Wilson. Op. cit., p. 335

37. Garner, R. Environmental politics. London: Harvester Wheatsheaf. 1996. Citado por: Ibid., p. 335.

38. Bromley, D.W., editor. The handbook of environmental economics. Oxford: Blackwell. Citado por: Ibíd., p. 335.

39. La economía ecológica examina la contribución del sistema económico en la formalización de la desigualdad e inequidad social y la degradación ambiental. 
Revista Luna Azul - ALGUNAS REFLEXIONES SOBRE EL ANÁLISIS TERRIT... Página 17 de 17

Así se evalúa, entre otras cosas, el daño ecológico y social causado por el sistema económico. De esta manera, esta subdisciplina puede ser de gran utilidad para los académicos y actores que trabajan en el campo de la gestión ambiental, en la medida que ayudaría a clarificar cómo las diferentes personas involucradas en este ámbito están diferencialmente incorporadas en el sistema económico, y su compromiso con el desarrollo de actividades ambientalmente sustentables. JACOBS Michael. Economía Verde. Oxford: Blackwell. 1994.

40. Reichel_Dolmatoff, G. The forest within: The world-view of the Tukano Amazonian Indians. London: Themis Bocks. 1996. Citado por: Ibid. p. 335.

41. Palacio Germán., Ulloa Astrid, Editores. p. 151.

42. El autor Andrew Vayda en su texto "Progressive contextualization: Methods for research in human ecology" reconoce que los modelos de la ecología cultural, que tomaron como pilares para explicar las características sociales con base en los conceptos de la ecología, evidenciaron sus deficiencias, puesto que dentro de la ecología se estaban abandonando las nociones de estabilidad y clímax, para aceptar la idea de continuidad sobre la de unidades discretas de análisis (o ecosistemas). Ibíd., p. 132.

43. Wapner, P. Politics beyond the state: environmental activism and world civic politics. World Politics 47, 311-40. 1995. Raymond L. Bryant; Geoff A. Wilson. Op. Cit., p. 335.

Close Window 